\title{
Subnotificação de casos de tuberculose a partir da vigilância do óbito*
}

\author{
Renata Olívia Gadelha Romero ${ }^{1}$, Cláudia Maria Cavalcanti Ribeiro ${ }^{2}$, Lenilde Duarte de Sá3 \\ Tereza Cristina Scatena Villa ${ }^{4}$, Jordana de Almeida Nogueira ${ }^{5}$
}

\author{
* Artigo extraído da dissertação de \\ Mestrado "Uso do sistema de informação \\ sobre mortalidade para identificar a \\ subnotificação de casos de tuberculose" \\ apresentada ao Programa de Pós- \\ Graduação em Enfermagem da \\ Universidade Federal da Paraíba em 2014. \\ ${ }^{1}$ Enfermeira, Mestre em Enfermagem. \\ Enfermeira assistencial no Hospital Giselda \\ Trigueiro. Natal, RN, Brasil. E-mail: \\ renatajpm@hotmail.com. \\ ${ }^{2}$ Enfermeira. Gerente de Vigilância \\ Epidemiológica da Secretaria Municipal de \\ Saúde de João Pessoa. João Pessoa, PB, \\ Brasil. E-mail: claudiamcra@gmail.com. \\ ${ }^{3}$ Enfermeira, Doutora em Enfermagem. \\ Professora Associado da Universidade \\ Federal da Paraíba. João Pessoa, PB, Brasil. \\ E-mail: sa.lenilde@gmail.com. \\ ${ }^{4}$ Enfermeira, Doutora em Enfermagem. \\ Professora Titular da Escola de \\ Enfermagem de Ribeirão Preto da \\ Universidade de São Paulo. Ribeirão Preto, \\ SP, Brasil. E-mail: tite@eerp.usp.br. \\ ${ }^{5}$ Enfermeira, Doutora em Enfermagem. \\ Professora Associado da Universidade \\ Federal da Paraíba. João Pessoa, PB, Brasil. \\ E-mail: jalnogueira31@gmail.com.
}

Recebido: 17/08/2015.

Aceito: 16/02/2016.

Publicado: 30/06/2016.

Como citar esse artigo:

Romero ROG, Ribeiro CMC, Sá LD, Villa TCS Nogueira JA. Subnotificação de casos de tuberculose a partir da vigilância do óbito. Rev. Eletr. Enf. [Internet]. 2016 [acesso em: ___ ] 18:e1161. Disponível em: http://dx.doi.org/10.5216/ree.v18.37249.

\section{RESUMO}

Objetivou-se analisar as subnotificações de casos de tuberculose a partir de captação de óbitos registrados no Sistema de Informação de Mortalidade (SIM) entre 2007 a 2011. Estudo retrospectivo, realizado em municipio nordestino, que utilizou a técnica de associação probabilística entre os registros do SIM e Sistema de Informação de Agravos de Notificação (Sinan), por meio do software Reclink III. Calculou-se odds ratio (OR) dos indivíduos que morreram com tuberculose não notificados no Sinan, segundo características sociodemográficas. Do total de 147 óbitos registrados no SIM, 72,8\% não haviam sido notificados no Sinan. Mulheres tiveram mais chance que os homens de não serem notificadas $(O R=2,60)$, pessoas com idade inferior a 60 anos $(O R=1,86)$ e com menos de oito anos de estudo $(O C=4,42)$. Estratégias devem ser ressignificadas de modo a aumentar a captação de sintomáticos respiratórios, diagnosticar oportunamente os casos e evitar falhas no preenchimento dos registros.

Descritores: Tuberculose; Sistemas de Informação em Saúde; Notificação de Doenças.

\section{INTRODUÇÃO}

A persistência da tuberculose (TB) no cenário mundial é reflexo das iniquidades- desigualdades sociais e econômicas, ocasionando importante impacto na morbimortalidade, principalmente nos segmentos mais vulneráveis da população. 
Em 2014, foram notificados no mundo, 9,6 milhões de casos novos de tuberculose, sendo que $12 \%$ (1,2 milhões) eram soropositivas para o HIV. No mesmo periodo ocorreram 1,5 milhão de óbitos por tuberculose sendo que $\mathbf{4 0 0 . 0 0 0}$ foram em pessoas coinfectadas (TB/HIV). Um total de $80 \%$ dos casos concentram-se em 22 países pobres e emergentes, com especial incidência na África Subsaariana, onde são registrados quatro de cada cinco casos de tuberculose ${ }^{(1)}$.

Segundo estimativas da Organização Mundial de Saúde (OMS), em 2014, o Brasil registrou incidência de 44 casos novos por 100.000 habitantes e uma taxa de mortalidade de 2,6 óbitos por 100.000 habitantes. Embora na última década se observe redução dos coeficientes de incidência e mortalidade, o país concentra $35 \%$ dos casos notificados na região das Américas $^{(1)}$ e ainda não conseguiu alcançar a meta estipulada pela OMS de detectar pelo menos $90 \%$ dos casos da doença ${ }^{(2)}$. Questiona-se a dificuldade dos serviços de saúde para captação de casos novos, erro de diagnóstico, diagnóstico tardio, ausência de busca de casos em diferentes fontes de registro, atraso nas notificações e no processamento das informações ${ }^{(3-4)}$.

Dentre as estratégias para o alcance das metas globais pactuadas, se faz necessário integrar sistematicamente um conjunto de ações de vigilância, que identifique os pontos de estrangulamento entre o processo de captação e notificação dos casos novos da doença ${ }^{(3)}$.

A informação em saúde deve ter como finalidade reduzir as incertezas e a identificação de situações prioritárias com vistas a subsidiar um planejamento adequado para a execução de ações que condicionem a realidade às transformações necessárias. Os Sistemas de Informação em Saúde (SIS) congregam vários subsistemas, cujos componentes possibilitam a coleta, o processamento, a análise e a transmissão da informação necessária para se planejar, organizar, operar e avaliar os serviços de saúde ${ }^{(5)}$. São verdadeiros dispositivos de apoio ao processo decisório.

Entretanto, não basta a disponibilidade de sistemas e recursos informáticos, se não forem compreendidos e operados adequadamente. Os avanços advindos do conhecimento científico e tecnológico e o melhor entendimento acerca do processo saúde-doença e seus determinantes, ampliaram o foco de análise da saúde pública. Por sua vez, expandiu-se a capacidade de mensurar o estado de saúde da população, a eficácia e efeitos dos serviços, que se processam pelo uso de indicadores de morbidade, mortalidade, de cobertura, de acesso a serviços, qualidade da atenção, condições de vida e fatores ambientais, entre outros. Evidentemente que a qualidade do dado produzido é elemento essencial para que se produzam indicadores de saúde que possam cumprir, a contento, suas finalidades ${ }^{(6)}$.

No controle à tuberculose, os dados gerados pelo Sistema de Informação de Agravos de Notificação (Sinan), subsidiam o desencadeamento oportuno de intervenções, monitoramento e avaliação de processos $^{(7)}$. Engloba a notificação e acompanhamento dos casos, reunindo informações quantitativas e qualitativas que constituem base para o cálculo de indicadores epidemiológicos e operacionais ${ }^{(8)}$. No entanto, sua utilidade é mediada pela qualidade dos dados, precisão e completitude das informações.

Adicionalmente, informações epidemiológicas podem ser obtidas de outros sistemas de informação em saúde como o Sistema de Informação sobre Mortalidade (SIM). A análise das bases de dados do SIM e do 
Sinan permite identificar o perfil epidemiológico dos casos de TB e acompanhar o indivíduo em diferentes situações da doença por meio da comparação $(\text { linkage })^{(9)}$ entre os sistemas, possibilitando ainda análises complementares sobre a vigilância e atendimento dos casos. A ocorrência de casos não notificados no Sinan pode sugerir barreiras no acesso aos serviços de saúde e falhas na qualidade do sistema, uma vez que, provavelmente, o diagnóstico foi dado ao indivíduo em situação extrema - óbito, por exemplo, sem ter sido diagnosticado previamente pela rede de atenção à saúde, em especial a atenção primária ${ }^{(10-12)}$.

Reconhecendo que a subnotificação dos casos de tuberculose a partir da vigilância do óbito, caracteriza-se como importante marcador para avaliar a gravidade da endemia, retardo no diagnóstico e tratamento oportuno, este estudo teve como objetivo analisar as subnotificações de casos de tuberculose a partir de captação de óbitos registrados no Sistema de Informação de Mortalidade entre 2007 a 2011.

\section{MÉTODO}

Foi conduzido um estudo retrospectivo, cuja população constituiu-se de todos os óbitos que apresentaram como causa básica a TB, independente da forma clínica (Classificação Internacional de Doenças-CID versão 10 códigos A15.0 a A19), registrados no Sistema de Informação sobre Mortalidade (SIM) no período de 2007 a 2011, residentes em capital do nordeste do Brasil. Ainda agregou os dados do Sistema de Informação de Agravos de Notificação (Sinan) com o proposito de identificar os casos que foram subnotificados.

A escolha do período de investigação se deu em função da introdução da versão do Sinan Net, em 2007, e ao encerramento oportuno dos casos de tuberculose até 2011. Com o objeto de aumentar a especificidade dos registros, dados de 2005 e 2006, do Sinan, também foram analisados.

As bases de dados foram disponibilizadas pela Gerência de Educação na Saúde do município de João Pessoa - Paraíba, sendo processados em setembro de 2013.

O estudo foi realizado em duas etapas: vinculação de registros do Sinan (rotina sistemática desse sistema), para tratamento das duplicidades; e linkage entre o Sinan e o SIM, para a identificação de subnotificações de tuberculose a partir do SIM.

O software tabwin versão $3.6 \mathrm{~b}$ foi utilizado para a vinculação e/ou exclusão de registros do Sinan. A vinculação se deu quando houve duplo registro (caso notificado mais de uma vez pela mesma ou outra unidade de saúde, durante o mesmo tratamento). Esse procedimento vincula duas fichas de notificação, sendo eliminada as repetições. Nesses casos, mantiveram-se os primeiros registros notificados e/ou complementaram-se os dados da 1a notificação com os dados da 2a notificação e, posteriormente, se deu a exclusão da $2 \underline{\text { a }}$ notificação

Foram excluídas as duplicidades verdadeiras (o mesmo paciente foi notificado, durante o mesmo tratamento, mais de uma vez pela mesma unidade de saúde). A exclusão dos registros ocorreu quando houve duas ou mais notificações do mesmo paciente, durante o mesmo tratamento e com a mesma situação de encerramento. 
Para investigar e quantificar os casos não detectados ou ignorados pelo Sinan-TB, utilizou-se o método de linkage probabilístico de registros, através do software RecLink III. Esse método consiste na identificação de campos em comum tanto no Sinan-TB e no SIM, cujo objetivo visa identificar o quão provável um par de registro faz referência a um mesmo indivíduo ${ }^{(9)}$.

No linkage entre o SIM e o Sinan-TB foram realizados cinco passos de blocagem com a combinação dos campos. Iniciou-se com uma chave mais restrita e com posterior diminuição da restrição buscando-se minimizar a perda de pares, ou seja, a ocorrência de falso-negativo:

- Soundex (primeiro nome) + soundex (último nome) + sexo;

- Soundex (primeiro nome) + soundex (último nome);

- Soundex (primeiro nome) + sexo;

- Soundex (último nome) + sexo;

No quinto passo de blocagem, repetiu-se a combinação do primeiro passo, com um valor de score menor, aumentando a sensibilidade para encontrar novos pares. Em todos os passos foram empregados para comparação e cálculo de escores os campos nome do paciente, nome da mãe e data de nascimento. Foi realizada a revisão manual dos pares duvidosos ao final de cada passo por um único pesquisador, obedecendo aos seguintes critérios de desempate: nome do paciente, nome da mãe, data de nascimento e município de residência.

Optou-se por considerar par o registro pareado que permaneceu como dúvida após o processo de revisão manual, no sentido de minimizar a ocorrência de erros falso- negativos. Dessa forma, os resultados observados podem ser interpretados como uma estimativa conservadora da subnotificação de casos de TB.

Os escores superiores a 22,4 foram considerados pares verdadeiros enquanto que os inferiores a 9,7 não pares, permanecendo os escores intermediários como duvidosos. Optou-se por considerar par o registro pareado que permaneceu como dúvida após o processo de revisão manual, no sentido de minimizar a ocorrência de erros (falsos negativos). Dessa forma, os resultados observados podem ser interpretados como uma estimativa conservadora da subnotificação de casos de TB.

Durante esse processo, registros de óbitos foram associados a mais de uma notificação do mesmo individuo no Sinan-TB por serem registros duplicados, casos de recidiva ou reingresso após abandono. Com o intuito de eliminar as repetições de um mesmo caso, foram excluídas, da análise, as notificações com data de diagnóstico mais antiga.

Foram recalculadas as taxas de incidência de TB do município, acrescentando-se os casos que não haviam sido notificados no Sinan e foram recuperados na base de dados do SIM, eliminando-se os casos coincidentes entre as bases. Foi utilizada a estimativa populacional para os anos de 2007 a 2011.

Calculou-se a razão de chances (odds ratio bruto) dos indivíduos que morreram com tuberculose não notificados no Sinan, segundo características sociodemográficas e, construiu-se seus respectivos intervalos de confiança - IC para um nível de significância de 5\%, através do método de Wolf. Para verificar a significância estatística foi utilizado o teste de associação do qui-quadrado. 
A pesquisa foi submetida à apreciação do Comitê de Ética em Pesquisa do Hospital Universitário Lauro Wanderley da Universidade Federal da Paraíba, aprovada em 27 de agosto de 2013 com o protocolo de $\mathrm{n}^{\circ}$ 377.615.

\section{RESULTADOS}

No período de 2007 a 2011 foram notificados 2.184 casos de tuberculose no Sinan-TB. Deste total, 396 notificações foram em 2007, 446 notificações em 2008, 491 notificações em 2009, 416 notificações em 2010 e 435 notificações em 2011. Nesse mesmo período, 147 óbitos por tuberculose foram registrados no SIM. Ao realizar o linkage entre as duas bases de dados, 107 óbitos por tuberculose não constavam no Sinan-TB, apresentando um percentual de $72,8 \%$ de subnotificação de casos da doença (Figura 1).

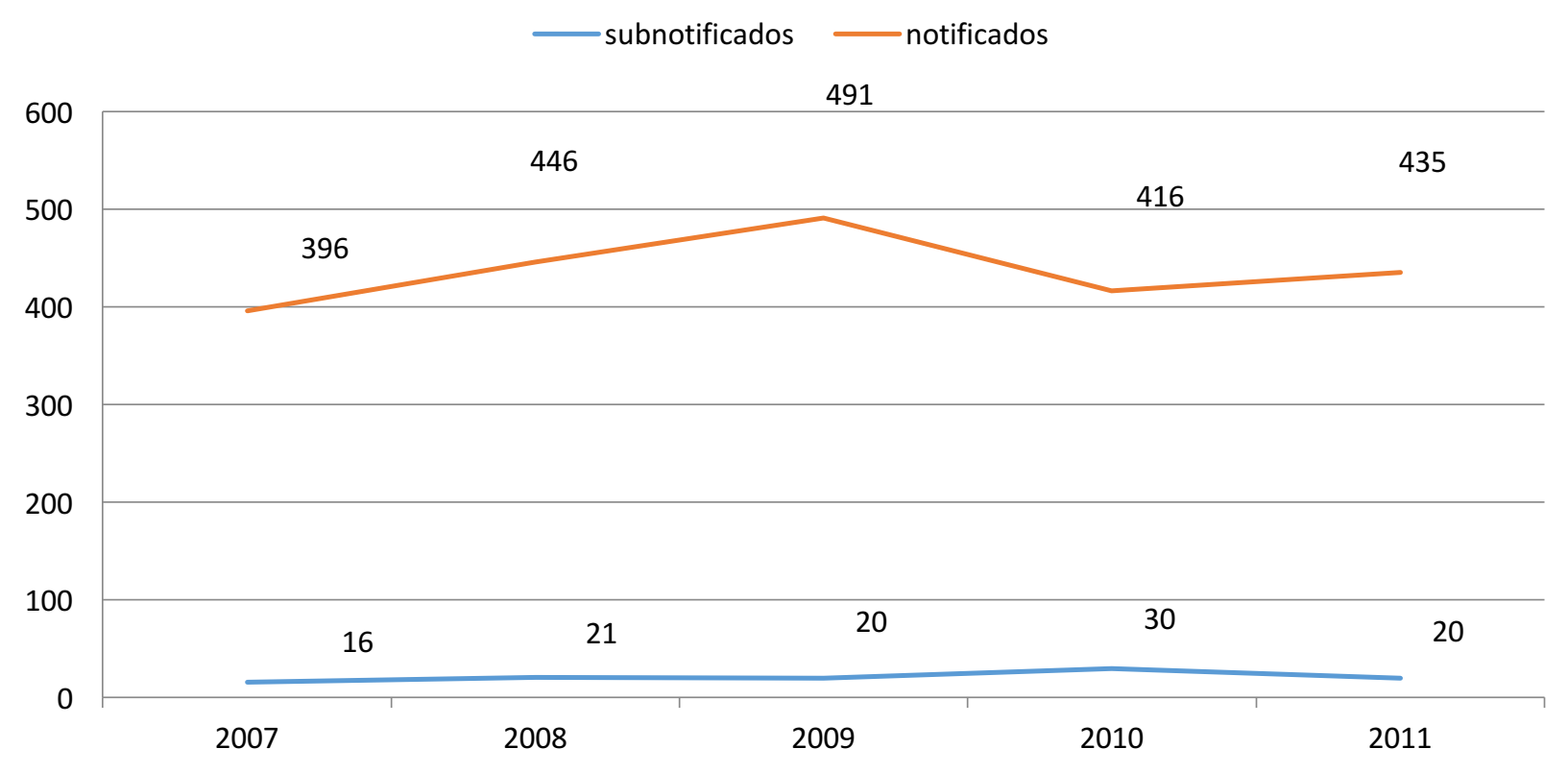

Figura 1: Casos de tuberculose notificados no Sinan e subnotificações identificadas a partir do SIM. João Pessoa, PB, Brasil, 2007 a 2011.

Em 2007, foram registrados 22 óbitos por tuberculose no SIM, sendo identificados 16 (72,7\%) subnotificações no Sinan; em 2008, 21 subnotificações (77,7\%), em 2009, 20 subnotificações (74,1\%), em 2010, 30 subnotificações (77,0\%) e em 2011, 20 subnotificações (64,5\%).

Dos casos notificados no SIM, no período em estudo, observa-se um $\mathrm{E}(\mathrm{x})$ (valor esperado) de registros de 21.4 (95\% IC=16.26-26.54) casos registrados anualmente, com 26.8 de variância amostral. No Sinan-TB, verifica-se um $\mathrm{E}(\mathrm{x})$ de $8(95 \% \mathrm{IC}=5.92-10.08)$ casos registrados anualmente, com 6.5 de variância amostral. Os valores de 21.4 e 8.0 são medidas centrais, mais próxima da verdade, porém não há garantia de que seja exatamente isso. A garantia é de que o risco relativo não está acima de 26.54 e 10.08 e nem abaixo de 16.26 e 5.92 para o SIM e Sinan-TB, respectivamente. Quanto mais estreito o intervalo de confiança, mais precisa é a estimativa. A precisão desses valores demonstra a capacidade de um resultado se repetir em diferentes 
medidas da mesma realidade.

Quanto ao coeficiente de incidência da doença antes e depois do relacionamento entre as bases de dados (Tabela 1), verifica-se que o acréscimo dos óbitos que não foram relacionados à base de dados do Sinan elevaria a taxa de notificação de casos novos, apresentando incremento da incidência de até $7,2 \%$.

Tabela 1: Coeficiente de incidência de tuberculose antes e depois da correção pelo relacionamento entre Sinan e SIM. João Pessoa, PB, Brasil, 2007 a 2011.

\begin{tabular}{llccccc}
\hline Ano & População & $\begin{array}{c}\text { Casos } \\
\text { Sinan }\end{array}$ & $\begin{array}{c}\text { Coeficiente incidência } \\
\text { (AC) }\end{array}$ & Sinan+SIM & $\begin{array}{c}\text { Coeficiente incidência } \\
\text { (DC) }\end{array}$ & $\begin{array}{c}\text { Incremento } \\
\text { Incidência }\end{array}$ \\
\hline 2007 & 683278 & 396 & 58,0 & 412 & 60,3 & 3,9 \\
2008 & 693082 & 446 & 64,4 & 467 & 67,4 & 4,6 \\
2009 & 702234 & 491 & 69,9 & 511 & 72,8 & 4,1 \\
2010 & 725515 & 416 & 57,3 & 446 & 61,5 & 7,2 \\
2011 & 733155 & 435 & 59,3 & 455 & 62,1 & 4,6 \\
\hline
\end{tabular}

AC: Antes da Correção; DC: Depois da Correção.

A subnotificação dos casos de TB a partir dos registros dos óbitos mostrou um perfil diferenciado entre os grupos populacionais (Tabela 2). Do total de óbitos ( $n=147$ ), 113 foram do sexo masculino, sendo que deste, 78 (69,0\%) não foram previamente notificados no Sinan. Embora a ocorrência das subnotificações tenha sido inferior entre as mulheres, verificou-se neste grupo, que o percentual proporcional de subnotificação dos casos foi superior a dos homens. Mulheres tiveram mais chance de não serem notificadas no Sinan em comparação aos homens $(O R=2,60)$.

Tabela 2: Odds ratio dos casos de óbito por tuberculose não-notificados no Sinan (2007-2011), segundo características sociodemográficas. João Pessoa, PB, Brasil, 2007 a 2011.

\begin{tabular}{|c|c|c|c|c|c|}
\hline Variáveis & Total & Não notificados - n (\%) & Odds bruto & IC 95\% & $p$-valor \\
\hline \multicolumn{6}{|l|}{ Sexo } \\
\hline Masculino & 113 & $78(69,0)$ & 1 & - & 0,062 \\
\hline Feminino & 34 & $29(85,3)$ & 2,60 & 0,$93 ; 7,29$ & \\
\hline \multicolumn{6}{|l|}{ Faixa Etária } \\
\hline$<60$ anos & 93 & $72(77,4)$ & 1,86 & 0,$89 ; 3,90$ & 0,098 \\
\hline 60 anos ou mais & 54 & $35(64,8)$ & 1 & - & \\
\hline \multicolumn{6}{|l|}{ Escolaridade * } \\
\hline Nenhuma & 19 & $5(26,3)$ & 0,40 & - & 0,196 \\
\hline$<8$ anos de estudo & 69 & $55(79,7)$ & 4,42 & 1,$44 ; 13,53$ & 0,006 \\
\hline$\geq 8$ anos de estudo & 17 & $8(47,0)$ & 1 & 0,$1 ; 1,62$ & \\
\hline \multicolumn{6}{|l|}{ Raça/Cor } \\
\hline Branca & 32 & $27(84,4)$ & 2,36 & 0,$84 ; 6,64$ & 0,096 \\
\hline Não branca & 115 & $80(69,6)$ & 1 & - & \\
\hline
\end{tabular}

* Casos sem informação sobre escolaridade foram excluidos.

Quanto a faixa etária, pessoas com idade inferior a 60 anos tiveram 1,86 vezes mais chance de não serem notificados quando comparados aos idosos. Apresentar menos de oito anos de estudo representou 4,42 vezes a chance de não ser notificado em relação a oito anos ou mais de estudo ( $p$-valor $<0,05)$. Não ter escolaridade representou fator de proteção para notificação $(O R=0,40)$.

No que diz respeito à variavel raça/cor, 115 (78,2\%) dos casos de tuberculose subnotificados no Sinan 
foram da cor não branca. Embora a ocorrência de óbitos tenha sido inferior nas pessoas de cor branca, este grupo apresentou maior chance de não ser notificado $(O R=2,36)$.

\section{DISCUSSÃO}

Os dados encontrados na pesquisa mostram que, entre os casos de óbitos notificados no SIM, 72,8\% não tiveram acesso ao diagnóstico da tuberculose em tempo oportuno. A correção do coeficiente de incidência da tuberculose no periodo analisado, a partir da inclusão dos novos casos da doença identificados por meio da vigilância do óbito, representou em média um acréscimo de 4,88\% nesse indicador.

A notificação permite refazer o caminho percorrido pelo paciente, em diferentes situações da doença, permite identificar possíveis fragilidades na organização do cuidado a esses usuários e possibilita rastrear oportunidade de transmissão(11-12). Considerando que o óbito é o desfecho mais crítico para os casos de tuberculose, o desconhecimento de sua ocorrência pelo setor de vigilância e a subnotificação dos casos no sistema de informação retratam fragilidades na detecção dos casos da doença.

Pesquisas realizadas no Brasil $^{(8,10-12)}$ retrataram subnotificações em, aproximadamente, metade dos óbitos com menção de TB, resultado semelhante ao relatado nesse estudo. Tal fragilidade sinaliza deficiências na qualidade e oportunidade de acesso dos usuários aos cuidados em saúde, as quais podem estar associadas a fatores interdependentes de cunho individual, social e programática. A perspectiva individual e social envolve percepção de risco, nível de conhecimento do processo saúde e doença, situação econômica, as relações de gênero e geracionais, valores culturais, acesso a recursos materiais e capacidade de receber informações, metabolizá-las e poder incorporá-las a mudanças práticas na vida cotidiana ${ }^{(13)}$. Neste caso, o desconhecimento sobre a doença e sua gravidade, dificuldades de acesso (cultural, geográfico, econômico) implicaria na busca tardia por serviços de saúde, em diagnóstico hospitalar ou no momento do óbito.

O componente institucional ou programático conecta os componentes individual e social. Consiste no enfrentamento do problema pelas autoridades locais, planejamento e gerenciamento das ações, capacidade de resposta das instituições envolvidas, financiamento adequado e estável. Se estende ao acesso aos serviços de saúde, qualidade da atenção, implantação e implementação de tecnologias de cuidado que propiciem a existência de contextos sociais favorecedores à adoção de medidas de prevenção(13).

Deve-se considerar, portanto, que a subnotificação dos casos de TB representam um estado de alerta aos gestores locais, pois sinaliza retardo no diagnóstico e tratamento, concorrendo para a inexpressividade das ações de promoção e prevenção na comunidade. O problema também enuncia deficiência nas atividades de busca de sintomáticos respiratórios, na identificação de casos suspeitos e na investigação de contatos.

Consensualmente, a tuberculose é mais frequente entre os homens do que entre as mulheres, assim como a ocorrência das subnotificações da doença ${ }^{(14-15)}$. Nesse estudo, as mulheres tiveram 2,60 vezes mais chance de não serem notificadas com a doença no Sinan em relação ao sexo masculino. Existe uma sensibilidade mais elevada na detecção do bacilo de Koch nas amostras de escarros provinientes do sexo 
masculino, o que por outro lado, constituiria um sinal de alerta para a subestimação de casos da doença no sexo feminino uma vez que esse recurso diagnóstico é menos sensivel ${ }^{(16)}$.

Quanto à faixa etária, pessoas com idade inferior a 60 anos tiveram mais chance de subnotificação da tuberculose do que os idosos. Este achado contraria estudos realizados em municípios da região sudeste do Brasil e da região central da Itália ${ }^{(14-15,17)}$, em que a proporção de indivíduos não notificados foi superior entre as pessoas mais velhas. Tal divergência pode estar associada ao modelo de organização da rede de atenção à saúde. Regiões que privilegiam a Estratégia de Saúde da Familia (ESF) como porta de entrada ao sistema de saúde, associam ao seu perfil de utilização, maior proporção de usuários idosos do que em áreas descobertas por esta estratégia ${ }^{(18)}$. Este pode ser um diferencial dos resultados encontrados neste estudo, em que predomina a ESF como modelo de atenção à saúde.

A chance de não ser notificado quando o indivíduo apresentava menos oito anos de estudo foi significativamente maior ( 4,42 vezes) do que a chance de não ser notificado entre os que tinham mais de oito anos de estudo. A baixa escolaridade reafirma a estreita relação entre o risco de adoecimento a estratos sociais com menor grau de instrução, os quais, por conseguinte, interferem no processo de suspeição e identificação da doença ${ }^{(19-21)}$. Estudo realizado no Rio de Janeiro mostrou que 58,4\% da amostra tinha menos de oito anos de estudo, sinalizando que o nível de instrução insuficiente dos clientes pode ter contribuído para o menor grau de percepção da doença ${ }^{(8)}$.

O maior percentual na ocorrência da subnotificação dos casos de tuberculose ser mais expressiva nas pessoas com baixa escolaridade pode estar relacioanda a diferença na oferta, no acesso aos serviços de saúde e na obtenção do diagnóstico precoce. Ainda, mesmo em estágio avançado da doença, indivíduos com melhor condição social podem tem maior acesso ao diagnóstico, contrariamente a situação encontrada na população com baixa condição social, que, talvez, nem no óbito seja confirmado o diagnóstico da doença ${ }^{(8,14)}$.

Em relação à raça, a chance de subnotificação foi 2,36 vezes maior entre os de cor branca. Estima-se que os brancos têm chances aumentadas em $3 \%$ de consumir serviços de saúde no Brasil ${ }^{(21)}$, além de que seus favorecimentos sociais os levam a dispor de melhores recursos na área da saúde. Neste caso, a subnotificação poderia estar associada à maior procura por serviços particulares, que por vezes desconhecem a política de controle da tuberculose ou não reconhecem nestes clientes, favorecidos economicamente, a possibilidade de adoecimento pela doença.

Há de se considerar ainda que a falta de resposta dos serviços de atenção básica pode ocasionar a busca por unidades de emergência e especializada. Embora no município estudado a cobertura populacional da Estratégia Saúde da Família seja de 84\%, estes serviços apresentam limitações para atuar como porta de entrada no que diz respeito à suspeição e confirmação diagnóstica da doença. Hospitais gerais/privados, policlínicas, consultórios médicos particulares e referência especializada para atendimentos de casos de TB permanecem como principal serviço diagnosticador ${ }^{(21-23)}$. Circunstancialmente, a subnotificação estaria relacionada àqueles casos que, mesmo tendo preenchido os critérios de definição de caso e sido identificado pelo profissional de saúde, não foi notificado ao serviço de saúde pública ou não foi notificado em tempo 
oportuno $^{(8)}$.

Implantar núcleos de vigilância hospitalar e avaliar e monitorar as rotinas de notificação pelos serviços privados seriam alternativas possíveis para induzir o desencadeamento de ações de vigilância, em especial no resgate de casos não notificados a autoridade sanitária. A vigilância da tuberculose pode ser útil para aumentar a completude dos sistemas de informação, resgatar e investigar a situação de encerramento dos casos, diminuir as subnotificações, aumentar a sensibilidade do Sinan e do SIM, avaliar a qualidade das declarações de óbito, supervisionar a vigilância epidemiológica dos estabelecimentos de saúde, resgatar o exame de contatos ainda não avaliados ${ }^{(11-12)}$.

\section{CONCLUSÃO}

Os resultados encontrados sinalizam fragilidades na coordenação de cuidado aos casos de tuberculose na rede de atenção à saúde do município. Denunciam baixa captação de sintomáticos respiratórios, erros de conduta clínica, diagnóstico tardio, falhas no preenchimento dos registros. Por sua vez, a maior expressividade de casos subnotificados na população do sexo feminino e com baixa escolaridade, sugerem barreiras no acesso deste grupo na busca e obtenção de serviços básicos de saúde.

Um outro ponto que merece destaque, reporta-se a qualidade no processo de produção das informações. Deficiências no preenchimento da ficha de notificação, inobservância dos prazos de envio e fragilidade no fluxo de informação entre as unidades notificadoras e a vigilância epidemiológica, podem comprometer um monitoramento seguro da realidade apresentada.

Investimentos precisam ser feitos na área de vigilância para fortalecer as atividades desse serviço. 0 linkage é uma técnica acessível e de baixo custo que pode aprimorar os desempenhos dos sistemas através de informações adicionais e mais fidedignas e auxiliar a tomada de decisão. A qualificação dos técnicos para o manuseio de base de dados e um treinamento para os profisisonais quanto a qualidade no preenchimento no registro de notificação dos casos de tuberculose são algumas ações expressivas que podem minimizar ou corrigir falhas existentes.

\section{REFERÊNCIAS}

1. World Health Organization. Global tuberculosis report 2015 [Internet]. Geneva: WHO; 2015 [acesso em: 30 jun. 2016]. Disponível em: http://apps.who.int/iris/bitstream/10665/191102/1/9789241565059_eng.pdf.

2. Ministério da Saúde. Detectar, tratar e curar: desafios e estratégias brasileiras frente à tuberculose. Boletim Epidemiológico [Internet]. 2015 [acesso em: 30 jun. 2016];46(9). Disponível em:

http://u.saude.gov.br/images/pdf/2015/marco/25/Boletim-tuberculose-2015.pdf.

3. Lytras T, Spala G, Bonovas S, Panagiotopoulos T. Evaluation of Tuberculosis Underreporting in Greece through Comparison with Anti-Tuberculosis Drug Consumption. Dowdy DW, editor. PLoS One [Internet]. 2012 [acesso em: 30 jun. 2016];7(11):e50033. Disponível em: http://dx.plos.org/10.1371/journal.pone.0050033.

4. Oliveira LCS, Nogueira JA, Sá LD, Palha PF, Silva CA, Villa TC. A discursividade do sujeito sobre sentimentos associados ao enfrentamento da tuberculose. Rev. Eletr. Enf. [Internet]. 2015 [acesso em: 30 jun. 2016];17(1):12-20. Disponível em: http://dx.doi.org/10.5216/ree.v17i1.24523.

5. Marin HF. Sistemas de informação em saúde: considerações gerais. J Heal Informatics [Internet]. 2010 [acesso em: 30 jun. 2016];2(1):20-4. Disponível em: http://www.jhi-sbis.saude.ws/ojs-jhi/index.php/jhi-sbis/article/view/4. 
6. Lima CRA, Schramm JMA, Coeli CM, Silva MEM. Revisão das dimensões de qualidade dos dados e métodos aplicados na avaliação dos sistemas de informação em saúde. Cad Saude Publica [Internet]. 2009 [acesso em: 30 jun. 2016];25(10):2095-109. Disponível em: http://dx.doi.org/10.1590/S0102-311X2009001000002.

7. Malhão TA, Oliveira GP, Codennoti S, Moherdaui F. Avaliação da completitude do Sistema de Informação de Agravos de Notificação da Tuberculose, Brasil, 2001-2006. Epidemiol e Serviços Saúde [Internet]. 2010 [acesso em: 30 jun. 2016];19(3):245-56. Disponível em: http://dx.doi.org/10.5123/S1679-49742010000300007.

8. Pinheiro RS, Andrade VL, Oliveira GP. Subnotificação da tuberculose no Sistema de Informação de Agravos de Notificação (SINAN): abandono primário de bacilíferos e captação de casos em outras fontes de informação usando linkage probabilístico. Cad Saude Publica [Internet]. 2012 [acesso em: 30 jun. 2016];28(8):1559-68. Disponível em: http://dx.doi.org/10.1590/S0102-311X2012000800014.

9. Camargo Jr. KR, Coeli CM. Reclink: aplicativo para o relacionamento de bases de dados, implementando o método probabilistic record linkage. Cad Saude Publica [Internet]. 2000 [acesso em: 11 jul. 2016];16(2):439-47. Disponível em: http://dx.doi.org/10.1590/S0102-311X2000000200014.

10. Oliveira GP, Pinheiro RS, Coeli CM, Codenotti SB, Barreira D. Linkage entre SIM e SINAN para a melhoria da qualidade dos dados do Sistema de Informação da tuberculose: a experiência nacional. Cad. saúde colet., (Rio J.) [Internet]. 2010 [acesso em: 30 jun. 2016];18(1):107-11. Disponível em:

http://www.cadernos.iesc.ufrj.br/cadernos/images/csc/2010_1/artigos/Modelo Livro UFRJ 10-a.pdf.

11. Selig L, Kritski AL, Cascão AM, Braga JU, Trajman A, Carvalho RMG. Proposta de vigilância de óbitos por tuberculose em sistemas de informação. Rev Saude Publica [Internet]. 2010 [acesso em: 30 jun. 2016];44(6):1072-8. Disponível em: http://dx.doi.org/10.1590/S0034-89102010000600012.

12. Sousa LMO, Pinheiro RS. Óbitos e internações por tuberculose não notificados no município do Rio de Janeiro. Rev Saude Publica [Internet]. 2011 [acesso em: 30 jun. 2016];45(1):31-9. Disponível em: http://dx.doi.org/10.1590/S003489102011000100004.

13. Ayres JRCM. O conceito de vulnerabilidade e as práticas de saúde: novas perspectivas e desafios. In.: Czeresnia D, Freitas CM. Promoção da saúde: conceitos, reflexões, tendências. 2a ed. Rio de Janeiro: Fiocruz; 2009. p. 117-39. 14. Lindoso AABP, Waldman EA, Komatsu NK, Figueiredo SM, Taniguchi M, Rodrigues LC. Perfil de pacientes que evoluem para óbito por tuberculose no município de São Paulo, 2002. Rev Saude Publica [Internet]. 2008 [acesso em: 30 jun. 2016];42(5):805-12. Disponível em: http://dx.doi.org/10.1590/S0034-89102008000500004.

15. Coelho AGV, Zamarioli LA, Perandones CA, Cuntiere I, Waldman EA. Características da tuberculose pulmonar em área hiperendêmica: município de Santos (SP). J Bras Pneumol [Internet]. 2009 [acesso em: 30 jun. 2016];35(10):9981007. Disponível em: http://dx.doi.org/10.1590/S1806-37132009001000009.

16. Allotey P, Gyapong M. Gender in tuberculosis research. Int J Tuberc Lung Dis. 2008 [acesso em: 30 jun. 2016];12(7):831-6. Disponível em:

http://www.ingentaconnect.com/content/iuatld/ijtld/2008/00000012/00000007/art00026.

17. Melosini L, Vetrano U, Dente FL, Cristofano M, Giraldi M, Gabbrielli L, et al. Evaluation of underreporting tuberculosis in Central Italy by means of record linkage. BMC Public Health [Internet]. 2012 [acesso em: 30 jun. 2016];12(1):472. Disponível em: http://dx.doi.org/10.1186/1471-2458-12-472.

18. Capilheira MF, Santos IS. Fatores individuais associados à utilização de consultas médicas por adultos. Rev Saude Publica [Internet]. 2006 [acesso em: 30 jun. 2016];40(3):436-43. Disponível em: http://dx.doi.org/10.1590/S003489102006000300011.

19. Bowkalowski C, Bertolozzi MR. Vulnerabilidades em pacientes com tuberculose no distrito Sanitário de Santa Felicidade - Curitiba, PR. Cogitare Enferm [Internet]. 2010 [acesso em: 30 jun. 2016];15(1):92-9. Disponível em: http://dx.doi.org/10.5380/ce.v15i1.17178.

20. Ximenes RAA, Albuquerque MFPM, Souza WV, Montarroyos UR, Diniz GTN, Luna CF, et al. Is it better to be rich in a poor area or poor in a rich area? A multilevel analysis of a case-control study of social determinants of tuberculosis. Int J Epidemiol [Internet]. 2009 [acesso em: 30 jun. 2016];38(5):1285-96. Disponível em:

http://dx.doi.org/10.1093/ije/dyp224.

21. Trigueiro DRSG, Nogueira JA, Monroe AA, Sá LD, Anjos UU, Villa TCS. Determinantes individuais e utilização dos serviços de saúde para o diagnóstico da tuberculose. Rev enferm UERJ [Internet]. 2011 [acesso em: 30 jun.

2016];21(3):371-8. Disponível em: http://www.facenf.uerj.br/v21n3/v21n3a16.pdf.

22. Oliveira AAV, Sá LD, Nogueira JA, Andrade SLE, Palha PF, Villa TCS. Diagnosis of tuberculosis in older people: 
barriers related to access to health services. Rev Esc Enferm USP [Internet]. 2013 [acesso em: 30 jun. 2016];47(1):14551. Disponível em: http://dx.doi.org/10.1590/S0080-62342013000100018.

23. Silva DM, Nogueira JA, Sá LD, Wysocki AD, Scatena LM, Villa TCS. Performance evaluation of primary care services for the treatment of tuberculosis. Rev Esc Enferm USP [Internet]. 2014 [acesso em: 30 jun. 2016];48(6):1041-50.

Disponível em: http://dx.doi.org/10.1590/S0080-623420140000700012. 